\title{
Emission of electromagnetic waves by proton beams in solar plasmas
}

\author{
J. I. Sakai and Y. Nagasugi
}

\begin{abstract}
Laboratory for Plasma Astrophysics Faculty of Engineering, Toyama University, 3190 Gofuku, Toyama 930-8555, Japan
e-mail: sakaijun@eng.u-toyama.ac.jp
\end{abstract}

Received 13 August 2007 / Accepted 7 September 2007

\section{ABSTRACT}

\begin{abstract}
Aims. We investigate the emission process of electromagnetic waves from proton beams propagating to a low-density region from a high-density region.

Methods. A two-dimensional, electromagnetic, relativistic Particle-In-Cell (PIC) code is used.

Results. It is found that the proton beams propagating to the low-density region are forced to move, together with the background electrons, to keep charge neutrality, resulting in the excitation of electrostatic waves: proton beam modes and Langmuir waves. In the early stage of electrostatic wave excitation, both $\mathrm{R}$ and $\mathrm{L}$ modes near the fundamental plasma frequency can be generated along a uniform magnetic field. It is also found that, in the late stage, the second harmonics of electromagnetic waves can be excited through the interaction of three waves. During these emission processes, proton beams can move along the magnetic field almost without losing their kinetic energy. The wave emission process by the proton beams may be applied to both the solar type III and type II radio bursts.
\end{abstract}

Key words. plasmas - radiation mechanisms: general - methods: numerical - Sun: radio radiation - Sun: flares

\section{Introduction}

A flare-associated solar type III burst occurs at the impulsive phase, which is more intense at meter wavelengths and may have a continuum attached to it (see for reviews, Goldman et al. 1986; Aschwanden 2004). It is believed that type III bursts are caused by mildly relativistic electrons $(\approx 10-100 \mathrm{keV})$ that are produced in impulsive solar flares, because bursts commonly occur in groups of ten or more, with a separation of seconds (Wild et al. 1963; McLean 1971).

A fundamental question concerning the origin of type III bursts is how they correlate with the mildly relativistic electrons and Langmuir waves produced from the electrons. Spacecraft measurements have verified the existence of both electron streams and Langmuir waves (Lin 1970). The next question concerning the origin of type III bursts is how the generated Langmuir waves can convert to the electromagnetic waves associated with the observed bursts. There are two classes of generation mechanisms for type III bursts: nonlinear wave-wave coupling processes (Lin et al. 1986) - the coalescence of two Langmuir waves, first proposed by Ginzburg and Zheleznyakov (1958) - and direct emission-mode conversion due to density inhomogeneity (Field 1956; Zheleznyakov 1970; Melrose 1980). Thejappa et al. (1993) evaluated emission mechanisms at $\omega_{\text {pe }}$ using ULYSSES observations and concluded that the direct-mode coupling mechanism is the most plausible process for type III bursts.

Recently, Sakai et al. (2005a) investigated the wave emission process of solar type III radio bursts from the magnetic reconnection region in a solar flare by using a two-dimensional, electromagnetic, relativistic Particle-In-Cell (PIC) code. They found that hot electron plasmas lead to the generation of Langmuir waves and the generated Langmuir waves can be converted to the electromagnetic waves (solar type III radio bursts) through the linear direct-mode conversion process. It is shown that the second harmonic emission is generated with time delay and with larger amplitude than for fundamental emission. However, their simulation shows that the electron beams do not propagate for a long time period along the magnetic field after emitting the waves. Therefore we need to consider some unknown physical processes for how the electron beams can propagate over a long time period.

In most strong flare events, the time profile of the prompt gamma-ray line emission caused by energetic protons is observed to be very similar to the profile of the bremsstrahlung hard X-rays emitted by energetic electrons (Aschwanden 2002). This suggests that the acceleration and propagation of the flareaccelerated protons and electrons are closely related. The most typical event among them is the 1980 June 7 flare observed by the SMM (Forrest \& Chupp 1983). This flare was explained by the current loop coalescence model (Tajima et al. 1982; Sakai \& Ohsawa 1987; Sakai \& De Jager 1996). Brown et al. (2000) suggest that hard X-rays in the flare impulsive phase are emitted by a neutralized proton beam due to the heating of the electrostatically dragged electrons.

Saito \& Sakai $(2004 \mathrm{a}, \mathrm{b})$ investigated the coalescence process of two parallel current loops with co-helicity by using twodimensional, electromagnetic, relativistic PIC code. They found that in a later stage of the two current loops' coalescence, fast magnetosonic waves are generated as a result of the coalescence rebounding and they then develop into shock waves. They also found that the protons can be promptly accelerated by the surfatron acceleration mechanism near the fast magnetosonic shock front (Sagdeev \& Shapiro 1973; Katsouleas \& Dawson 1983; Ohsawa 1985; Ohsawa \& Sakai 1987). Recently, Saito \& Sakai (2006) found that, during the formation of current sheet, protons 
can be accelerated through the surfatron mechanism by the fast magnetosonic shock waves. They point out (Sakai et al. 2005b, 2006) that during the type II radio bursts that can be produced from the fast magnetosonic shock waves, protons are also accelerated by the surfatron acceleration mechanism.

Motivated by the above studies, we investigated the electromagnetic wave emission process from the proton beams produced by the fast magnetosonic shock waves. The proton velocity accelerated by the surfatron mechanism reaches about $\left(m_{\mathrm{p}} / m_{\mathrm{e}}\right)^{1 / 2}\left(M_{\mathrm{A}}-1\right)^{3 / 2} V_{\mathrm{A}}\left(M_{\mathrm{A}}\right.$ is Alfvén Mach number $)$, which becomes comparable to $c / 3$. We assumed the presence of proton beams with about $\left(m_{\mathrm{p}} / m_{\mathrm{e}}\right)^{1 / 2} V_{\mathrm{A}}$ propagating along the uniform magnetic field from a high-density region. We found that the proton beams propagating to a low-density region from the highdensity region are forced to move together with the electrons to keep charge neutrality, resulting in the excitation of electrostatic proton beam modes, as well as Langmuir waves. In the early stage of the excitation of the beam modes, both $\mathrm{R}$ and $\mathrm{L}$ modes near the fundamental plasma frequency can be emitted along a uniform magnetic field. We also found that in the late stage the second harmonics of the electromagnetic waves can be excited through the three waves' interaction. During these emission processes, proton beams can move along the magnetic field almost without losing their kinetic energy. In Sect. 2 we present simulation model and simulation results. In Sect. 3 we summarize our results and discuss applications to solar radio bursts.

\section{Simulation model and simulation results}

We assumed that proton beams are generated in a high-density region by the surfatron acceleration process in the fast magnetosonic shock front. We investigated the emission process of electromagnetic waves when the proton beams escape from the shock region and propagate along a uniform magnetic field ( $y$ direction) from the high to the low density region. We used 2D3V, fully relativistic electromagnetic PIC code, modified from 3D3V TRISTAN code (Buneman 1993). The system size is $L_{x}=10 \Delta, L_{y}=6000 \Delta$, where $\Delta(=1)$ is the grid size. This means that the simulation results correspond to a $1 \mathrm{D} 3 \mathrm{~V}$ simulation including only the y spatial coordinate. The free boundary condition for the $y$ direction is imposed on particles and fields, while the periodic boundary condition is imposed in the $x$ direction. The background plasma density decreases linearly in the negative $y$-direction as shown in Fig. 1. The high density at $y=6000$ is 300 particles in a cell, while the low-density region at $y=0$ is 100 particles in a cell. The ratio between proton beam and background protons is about $5 / 300=0.017$ at $y=6000$. The reason we chose this ratio is that this value is a typical value for protons that can be accelerated by the surfatron mechnism near the shock front. There are about 12 million uniformly distributed electron-proton pairs in the system, which are keeping the charge neutrality. The region where proton beams were added in the system is also initially kept the charge neutrality. Those proton beams with velocity of $-0.27 c$ were added near the high-density region $(5000<y<6000)$, where the average numbers of proton beams in a cell is about 5 particles. The proton velocity corresponds to about $30 V_{\mathrm{A}}$ where the Alfvén velocity is $0.0093 \mathrm{c}$. The proton temperature is equal to the electron temperature. Other parameters are as follows: the time step $\omega_{\mathrm{pe}} \Delta t=0.05$, mass ratio $m_{\mathrm{i}} / m_{\mathrm{e}}=1836.0$, Debye length $v_{\mathrm{th}, \mathrm{e}} / \omega_{\mathrm{pe}}=1.0 \Delta$, and the collisionless skin depth $c / \omega_{\mathrm{pe}}=10 \Delta$. The physical quantities associated with the magnetic field $B_{0}$, like the ratio of $\omega_{\text {ce }}$ to $\omega_{\text {pe }}$ at $y=0$, plasma beta, electron Larmor radius, and ion Larmor radius, are $0.4,1 / 8,2.5 \Delta$, and $107.1 \Delta$, respectively.

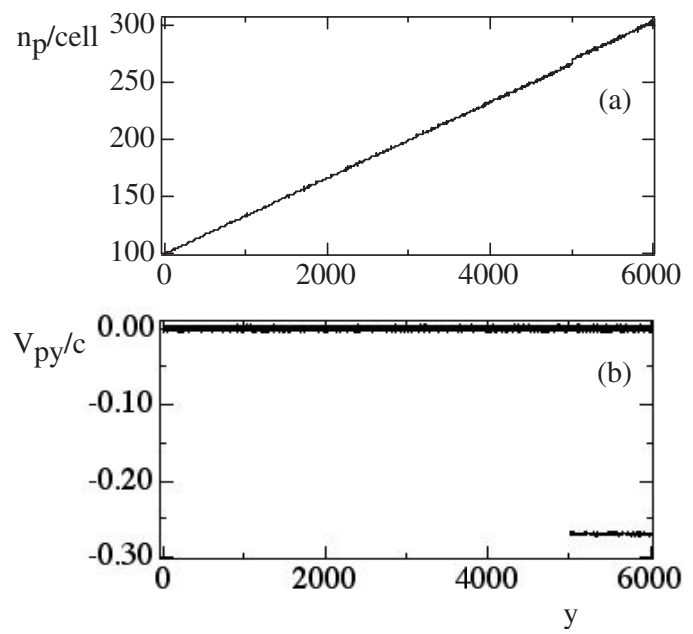

Fig. 1. a) The initial proton density distribution, and b) the initial proton velocity phase-space $\left(V_{\mathrm{py}}-y\right)$ plot. The proton beam with a negative velocity of $0.27 \mathrm{c}$ in the $y$-direction is located at $5000<y<6000$ in the high-density region.

First of all, we show how the proton beams propagate along a uniform magnetic field. In Fig. 2a we show the initial electron velocity phase $\left(V_{\text {ey }}-y\right)$ plot, and in Fig. $2 \mathrm{~b}$ the initial proton phase $\operatorname{space}\left(V_{\mathrm{py}}-y\right)$ plot. In Figs. $2 \mathrm{c}$ and $2 \mathrm{~d}$ the electron and proton velocity phase space plots at $\omega_{\mathrm{pe}} t=1500$ are shown. As seen in Fig. 2d, the proton beams propagate in the negative $y$ direction almost without losing their kinetic energy, while the some background electrons tend to move together with proton beams to keep the charge neutrality and some fraction of the electrons are accelerated in the front of the proton beams.

To understand what is happening during the interaction between proton beams and background electrons, we analyzed the electric fields by using two-dimensional space and time Fourier transformation during the early phase of the interaction. The spatial structures of the electrostatic electric field $E_{y}$ and $E_{z}$ at $\omega_{\text {pe }} t=400$ are shown Figs. 3a and 3b, respectively. Figure 3c shows the dispersion relation of the electrostatic field $E_{y}$ that was obtained from a space-time Fourier transformation taken in the region of $2800<y<4848$ and $400<\omega_{\mathrm{pe}} t<502.4$. The proton beam mode and Langmuir waves are excited. Figure $3 \mathrm{~d}$ shows the dispersion relation of the electromagnetic waves $E_{z}$ obtained from a space-time Fourier transformation taken in the region of $2800<y<4848$ and $400<\omega_{\text {pe }} t<502.4$. The upper wave showing the R-mode and lower wave of the L-mode are observed in Fig. 3d. The cut-off frequencies of the R- and L-modes are given by the following expressions, respectively.

$$
\begin{aligned}
& \omega_{\mathrm{R}}=0.5 \omega_{\mathrm{ce}}\left[\left(1+4 \omega_{\mathrm{pe}}^{2} / \omega_{\mathrm{ce}}^{2}\right)^{1 / 2}+1.0\right] \\
& \omega_{\mathrm{L}}=0.5 \omega_{\mathrm{ce}}\left[\left(1+4 \omega_{\mathrm{pe}}{ }^{2} / \omega_{\mathrm{ce}}\right)^{1 / 2}-1.0\right]
\end{aligned}
$$

If we take the electron plasma frequency at the density of $n_{0}=$ 250 where the R- and L-modes are excited, we get $\omega_{\mathrm{R}}=1.8$ and $\omega_{\mathrm{L}}=1.4$. Here, the cut-off frequencies agree well with the values obtained from the simulation.

Next we investigated the wave emission process during the late phase of the interaction between the proton beams and the background electrons. In Figs. $4 a$ and $4 b$, we show the spatial structures of electrostatic electric field $E_{y}$ and $E_{z}$ at $\omega_{\mathrm{pe}} t=1250$, respectively. Figure $4 \mathrm{c}$ shows the dispersion relation of the electrostatic field $E_{y}$, which was obtained from a space-time Fourier 

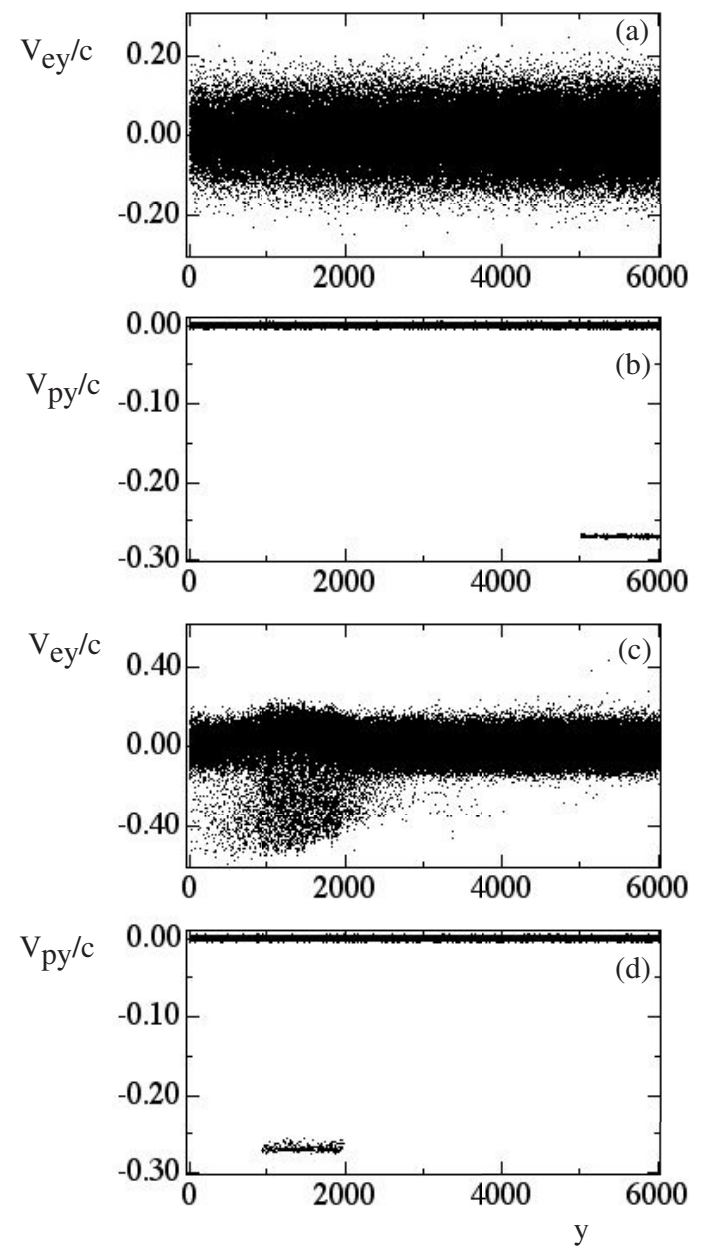

Fig. 2. a) The initial electron velocity phase space $\left(V_{\mathrm{ey}}-y\right)$ plot, b) the initial proton phase space $\left(V_{\mathrm{py}}-y\right)$ plot. and the electron $\left.\mathbf{c}\right)$ and proton velocity phase space d) plots at $\omega_{\text {pe }} t=1500$.

transformation taken in the region of $1904<y<6000$ and $1250<\omega_{\text {pe }} t<1352.4$. It is seen that the proton beam mode and Langmuir waves are excited. Figure $4 d$ shows the dispersion relation of the electromagnetic waves $E_{z}$ that was obtained from space-time Fourier transformation taken in the region of $1904<y<6000$ and $1250<\omega_{\mathrm{pe}} t<1352.4$. It is found that both forward and backscattered plasma waves are excited near the fundamental plasma frequency. It is also seen that second harmonic electromagnetic waves are excited to both directions. These second harmonic waves could be excited by the three waves' interaction $\left(\omega_{3}=\omega_{1}+\omega_{2}\right)$ in both positive and negative $y$-directions. The wave with frequency $\omega_{2}$ is the electrostatic wave shown in Fig. 4c, while the wave with frequency $\omega_{1}$ is the electromagnetic wave propagating backward.

In Fig. 5a we show the velocity distribution functions of the electron and proton at $\omega_{\mathrm{pe}} t=0$, while in Fig. 5b we show the velocity distribution functions of the electron and proton at $\omega_{\text {pe }} t=1500$. During the interaction between proton beams and background plasma, proton beams can propagate almost without damping and can emit the electromagnetic waves. The background electrons are accelerated to keep the charge neutrality, resulting in the excitation of beam modes, as well as Langmuir waves. The excited Langmuir waves can be transformed into the electromagnetic waves propagating both forward and backward.

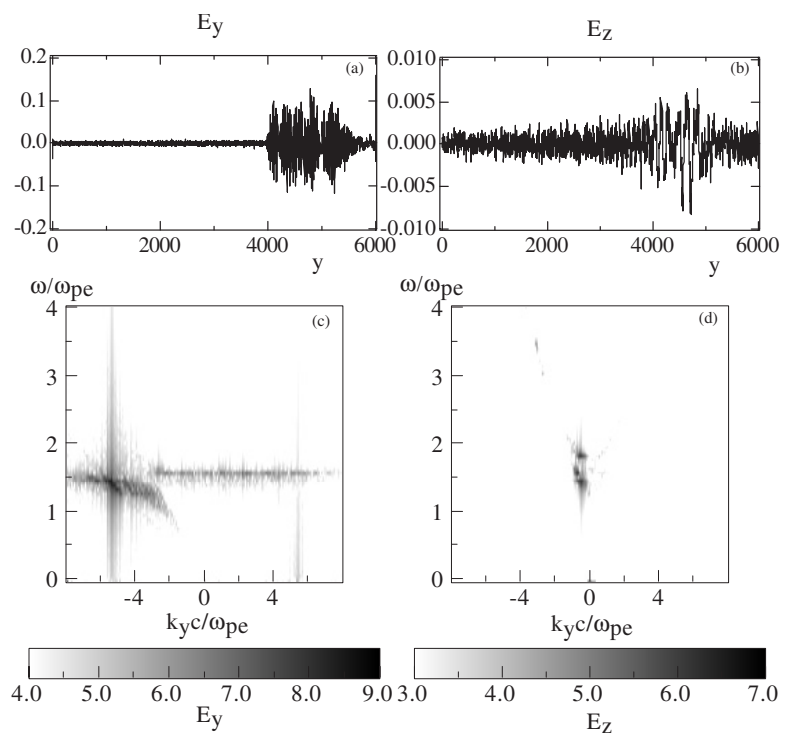

Fig. 3. a) The spacial structures of electrostatic electric field $E_{y}$ and b) $E_{z}$ at $\omega_{\text {pe }} t=400$. c) The dispersion relation of the electrostatic field $E_{y}$ that was obtained from space-time Fourier transformation taken in the region of $2800<y<4848$ and $400<\omega_{\mathrm{pe}} t<502.4$. The proton beam mode and Langmuir waves are excited. d) The dispersion relation of the electromagnetic waves $E_{z}$ that was obtained from space-time Fourier transformation taken in the region of $2800<y<4848$ and $400<$ $\omega_{\mathrm{pe}} t<502.4$. The upper wave showing the R-mode and lower wave of the L-mode are observed in $\mathbf{d}$ ).

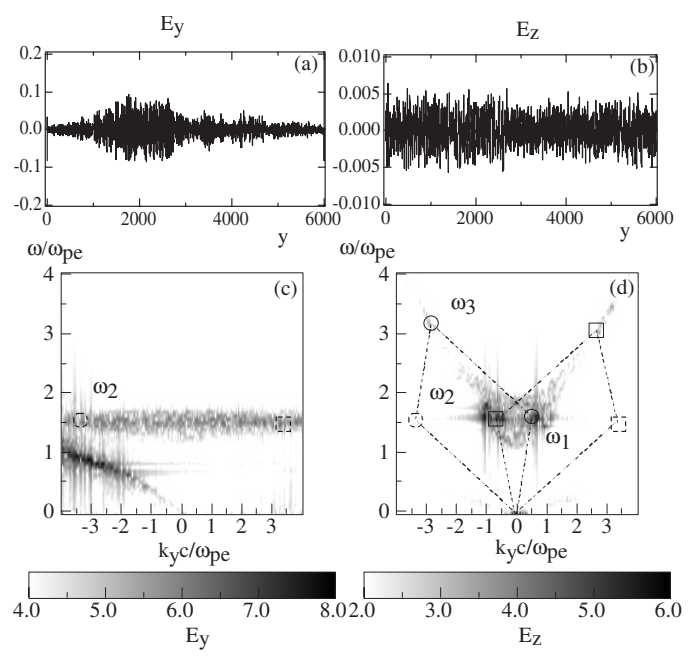

Fig. 4. a) The spacial structures of electrostatic electric field $E_{y}$ and b) $E_{z}$ at $\omega_{\mathrm{pe}} t=1250$. c) The dispersion relation of the electrostatic field $E_{y}$ that was obtained from space-time Fourier transformation taken in the region of $1904<y<6000$ and $1250<\omega_{\mathrm{pe}} t<1352.4$. The proton beam mode and Langmuir waves are excited. d) The dispersion relation of the electromagnetic wave $E_{z}$ that was obtained from spacetime Fourier transformation taken in the region of $1904<y<6000$ and $1250<\omega_{\mathrm{pe}} t<1352.4$. Second harmonic electromagnetic waves (solid circle and solid square in d)) are excited by the three waves' interaction are observed in $\mathbf{d}$ ).

\section{Conclusions}

We investigated the emission process of electromagnetic waves from proton beams by using two-dimensional, electromagnetic, relativistic PIC code. We found that the proton beams propagating to a low-density region from a high-density region are forced to move together with the background electrons to keep charge 
$\log f$
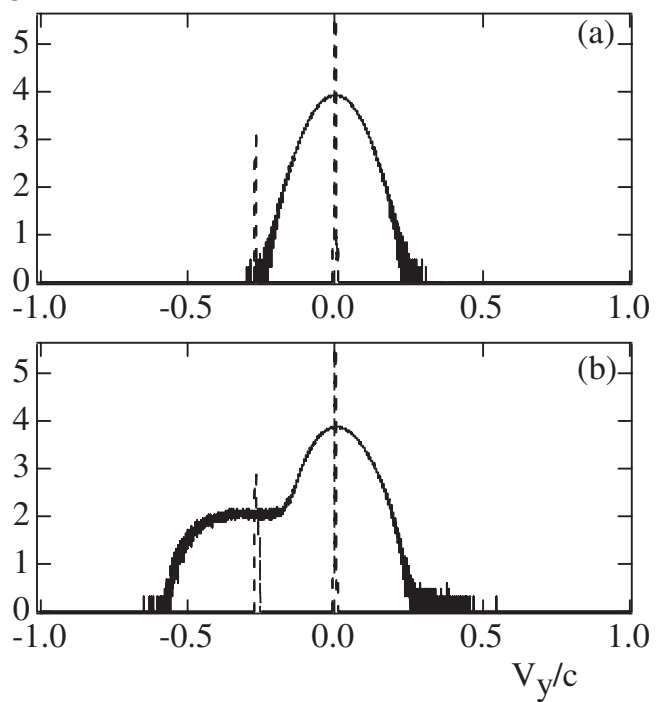

Fig. 5. a) The velocity distribution functions of the electron (solid line) and proton (dashed line) at $\omega_{\mathrm{pe}} t=0$. b) The velocity distribution functions of the electron (solid line) and proton (dashed line) at $\omega_{\mathrm{pe}} t=1500$.

neutrality, resulting in the excitation of electrostatic proton beam modes, as well as the acceleration of background electrons. In the early stage of the excitation of the beam modes, both $\mathrm{R}$ and $\mathrm{L}$ modes near the fundamental plasma frequency can be excited along the uniform magnetic field. We also found that in the late stage, the second harmonics of electromagnetic waves can be excited through the three waves' interaction. During these emission processes, proton beams can move along the magnetic field almost without losing their kinetic energy. The wave emission process by the proton beams may be applied to both the solar type III and type II radio bursts. The band splitting observed in the type II bursts may correspond to the R- and L-mode, while in the un-magnetized plasmas the band splitting effect disappears. Here we assumed that proton beams generated from the shock region propagate along a uniform magnetic field. In a subsequent paper we will investigate the effect of proton beams propagating obliquely to the magnetic field.

Acknowledgements. The authors thank the anonymous referee for useful comments.

\section{References}

Aschwanden, M. J. 2002, Space Sci. Rev., 101, 1

Aschwanden, M. J. 2004, in Physics of The Solar Corona (Springer-Verlag), Chap. 15,657

Brown, J. C., Karlicky, M., Mandzhavidze, N., \& Ramaty, R. 2000, ApJ, 541, 1104

Buneman, O. 1993, in Computer Space Plasma Physics, Simulation Techniques and Software, ed. H. Matsumoto, \& Y. Omura (Tokyo: Terra Scientific), 67 Field, G. B. 1956, ApJ, 124, 555

Forrest, D. J., \& Chupp, E. L. 1983, Nature, 305, 291

Ginzburg, V. L., \& Zheleznyakov, V. V. 1958, Soviet Astron. J., 2, 653

Goldman, M. V., \& Smith, D. F. 1986, Chap. 15 in Physics of the Sun, ed. P. A. Sturrock, T. E. Holzer, D. M. Mihalas, \& R. K. Ulrich (D. Reidel, Publ. Co.), 2, 325

Katsouleas, T., \& Dawson, J. M. 1983, Phys. Rev. Lett., 51, 392

Lin, R. P. 1970, Solar Phys., 12, 266

Lin, R. P., Levedahl, W. K., Lotko, W., Gurnett, D. A., \& Scarf, F. L. 1986, ApJ, 308,954

McLean, D. J. 1971, Austral. J. Phys., 24, 201

Melrose, D. B. 1980, Aust. J. Phys., 33, 121

Ohsawa, Y. 1985, Phys. Fluids, 28, 2130

Ohsawa, Y., \& Sakai, J. I. 1987, ApJ, 313, 440

Sagdeev, R. Z., \& Shapiro, V. D. 1973, JETP Lett., 17, 279

Sakai, J. I., \& de Jager, C. 1996, Space Sci. Rev., 77, 1

Sakai, J. I., \& Ohsawa, Y. 1987, Space Sci. Rev., 46, 113

Saito, S., \& Sakai, J. I. 2004a, ApJ, 616, L179

Saito, S., \& Sakai, J. I. 2004b, Phys. Plasmas 11, 2, 5547

Saito, S., \& Sakai, J. I. 2006, ApJ, 652, 793

Sakai, J. I., Kitamoto, T., \& Saito, S. 2005a, ApJ, 622, L157

Sakai, J. I., Mori, T., \& Saito, S. 2005b, A\&A, 442, 687

Sakai, J. I., Mori, T., Saito, S., Tanaka, Y., \& Aurass, H. 2006, A\&A, 454, 983

Tajima, T., Brunel, F., \& Sakai, J. I. 1982, ApJ, 245, L45

Thejappa, G., Lengyel-Frey, D., Stone, R. G., \& Goldstein, M. L. 1993, ApJ, 416,831

Wild, J. P., Smerd, S. F., \& Weiss, A. A. 1963, ARA\&A, 1, 291

Zheleznyakov, V. V. 1970, Radio Emission of the Sun and Planets (New York: Pergamon) 